\title{
MAC Support for High Density Wireless Sensor Networks
}

\author{
C. Taddia $\nmid$, N. Meratnia $\ddagger$ L.F.W. van Hoesel $\ddagger$, G. Mazzini $†$, P.J.M. Havinga $\ddagger$ \\ $\dagger$ ENDIF, University of Ferrara, Via Saragat 1, 44100 Ferrara, Italy. \\ $\ddagger$ Computer Science Department, University of Twente, Postbus 217, NL-7500 AE Enschede, The Netherlands. \\ E-mail: \{ctaddia, gmazzini\}@ing.unife.it, \{n.meratnia, 1.f.w.vanhoesel,p.j.m.havinga\}@utwente.nl
}

\begin{abstract}
Large scale and high density networks of tiny sensor nodes offer promising solutions for event detection and actuating applications.

In this paper we address the effect of high density of wireless sensor network performance with a specific MAC protocol, the Lightweight Medium Access Control (LMAC). We propose a power control support for the LMAC protocol to successfully cope with high density issue.

Experimental results show that our power control support significantly increases the number of nodes that can control a timeslot, without affecting other performance metrics such as the latency.
\end{abstract}

\section{INTRODUCTION}

It was not a long ago that the wireless sensor network (WSN) was considered as a cool technology only for the purpose of sensing and monitoring [1], [2]. Due to the recent advances in this technology, we now witness a shift in the application domains that WSN is used for. Sensing and monitoring have become fundamental features WSN should be able to provide in addition to other features such as event detection and actuating [3], to name but a few. Combining sensing, monitoring, event detection and actuation has provided a strong tool which has made new application domains to greatly benefit from WSN technology. One of such applications is forest fire management [4], which has great impact on community at large by providing a safer environment and offering security through on-time detection of possible fires and directions of fire spread and consequently generating early warnings as well as facilitating extinguishing the fire. Devastating fires destroy many forests every year across the glob, bring unrecoverable social, economical, and ecological damage, waste one of the great earth's resources, and threaten, harm, and affect lives.

Large scale and high density deployment of tiny sensor nodes equipped with various sensors such as smoke, temperature, gas (e.g. $N O_{x}$ and $C O_{x}$ ) that automatically form a network, wirelessly communicate with each other, collaboratively monitor the forest and cooperatively sense and measure various environmental parameters is essential for effective early fire detection. Moreover, fast, distributed, and in-network data processing is a must to be able to react appropriately upon detecting the fire. In addition, dynamic adaptation to continuously changing resources and requirements of the deployed network and its topology due to damaged nodes and arrival of new ones to allow increasing/decrising the coverage, and repair the connectivity of the network are quite crucial. Realizing an effective and efficient forest fire management application brings along notions of high density, heterogeneity, scalability, adaptability \& dynamic reconfigurability, and reliability to name but a few.

Our focus in this paper is on the issue of high density, which needs successful tackling of the following subjects, each of which is a researcher topic by itself:

- Power control: high density network requires mechanism to determine, set, and control nodes' transmission power to establish an always connected energy efficient network. The transmission range needs periodically to be updated depending on the nodes density to maintain the network connectivity.

- Wireless medium sharing: in a high density network the need for robust MAC protocols to share the wireless medium in a way that reduces the number of collisions while each node has a chance of transmitting its data is even more crucial than low density networks.

- Network topology control: in a high density network, topology control solutions are needed to determine the best place to locate sensor nodes, as well as to optimize the network performance.

- Autonomy and self-organizing: high density network even more than low density network need great investment in network configuration and maintenance unless sensor nodes themselves have an effective autonomy and self-stabilizing mechanism in place.

- Competition for limited resources: there is no doubt that network resources such as bandwidth are limited. Therefore, the competition between sensor nodes to use network resources increases as the nodes density increases.

- Data aggregation \& consensus: having many nodes monitoring a single area has the advantage that the overall performance of the network will not be affected by failure of a single node. Neighboring nodes that are close enough to observe the similar parameters could compensate for erroneous readings or missing data of a node. In addition the accuracy of the data gathering can be improved by having more readings and averaging them. However, this in turn requires reliable, fast, local, and distributed data processing which does not lead to extra overhead and reducing the performance of the network.

To more closely investigate the effects of high density on 
wireless sensor network performance, we consider the LMAC protocol [5], i.e., a time division multiple access (TDMA) based, energy efficient medium access control. LMAC strongly relies on the number of time slots, so to the density and connectivity of the network. High node density results in unavailability of free slots and consequently failure of the nodes to access the wireless medium. This has a great negative effect on the network performance at large. We, therefore, propose an adaptive power control mechanism to successfully cope with high density issue in wireless sensor networks.

The rest of the paper is organized as follows. Section II briefly introduces LMAC protocol and its principles. Our power control support mechanisms are presented in Section III, which is followed by the results of our extensive experiments and evaluations in Section IV. An overview of the related work is given in Section V. Section VI concludes the paper and provides an insight on our plans for future work.

\section{LMAC PROTOCOL}

The Lightweight Medium Access Control (LMAC) is an energy-efficient medium access protocol designed for wireless sensor networks. It uses a single frequency channel and the wireless medium is shared among the nodes with a time division multiple access (TDMA) approach. Scheduled protocols are often used for WSN because of their energy efficiency. Since the bandwidth is pre-allocated to individual nodes, there is no energy wasted on collisions due to channel contention. Among scheduled protocols, TDMA seems to be more suitable for WSN since it can support low duty cycle operations and it avoids overhearing the channel by turning off the radio during the slots of other nodes. Like other time-scheduled MAC protocols, LMAC considers time to be divided into slots which are further organized into periodic frames. Each node communicating data to other nodes has to control a timeslot of the frame, therefore each node has the possibility to access the wireless medium once in each frame, during its own slot.

Although reserving a timeslot for each node is a rather simple timeslot allocation, since WSN are typically composed of a large number of nodes, this would increase the latency of communication before nodes get the opportunity to transmit. For this reason, the frame interval should be kept as small as possible and the slots should be reused as much as possible.

LMAC defines timeslot assignment in a fully distributed manner, based on only local information and without the need of any central authority to allocate the timeslots to the nodes. Furthermore, it allows the reuse of the slots within the network, without causing interference between nodes that own the same timeslot in the frame. This is because it ensures that a timeslot is only reused by nodes that are, at least, two hops away.

The LMAC protocol works as follows. At the beginning of each timeslot, each node owning this slot broadcasts a Control Message (CM) containing information such as its identity, the destination address, and synchronization details. During this operation all nodes must to be on and listen at the beginning of each timeslot in order to receive CM. After this, the source nodes can send the actual data, during which the intended destination nodes remain on in order to receive data while all the other other nodes can turn off their transceiver for the rest of the slot for the purpose of energy saving. The CMs are also used by LMAC to implement the slot allocation algorithm. A CM contains also a vector, called OccupiedSlots, storing information about the slots occupied by the 2-hops neighbors. Each field of the OccupiedSlots vector represents a slot of the frame. Using the OccupiedSlots vector a node forwards information about those timeslots that it considers to be occupied by itself and its 1-hop neighbors. In this way, a node that does not yet control a slot knows the slots occupied in its 2-hop neighborhood (by performing OR operation between the OccupiedSlots vectors of all the received CM) and can consequently choose to control a slot among the free ones left after the OR operation. This method ensures that a timeslot is only reused after at least 2-hops. During the network set up, two or more nodes in a mutual range may choose simultaneously the same slot, and cause collisions in the wireless medium. Collisions are resolved thanks to another vector exchanged in the CM, namely the CollisionSlot. Each field of the CollisionSlot represents a slot of the frame. When a node detects that two or more of its neighbors use the same slot and therefore there will be a collision, it informs them by properly setting the CollisionSlot field corresponding to the slot in which it detects the collision. Consequently, nodes that receive $\mathrm{CM}$ containing collisions detected in their own slot immediately free their controlled slots and re-start the process of finding a free slot. The distributed algorithm for timeslot selection is shown in Figure 1. When there are no more free slots, the nodes that do not yet control a slot remain in an initialization state, and monitor periodically the channel to find an empty timeslot.

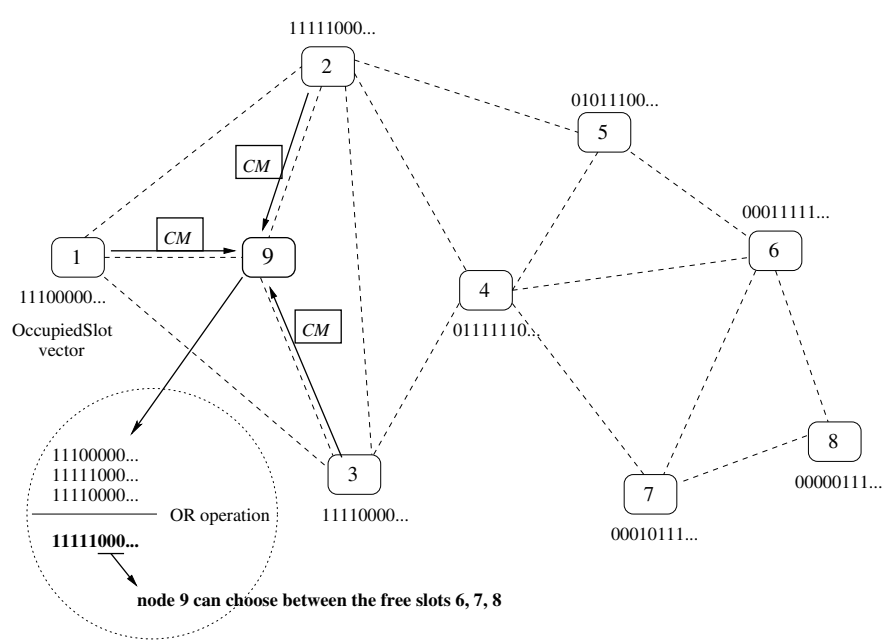

Figure 1. Slot allocation in LMAC protocol.

\section{POWER CONTROL SUPPORT FOR LMAC}

Dealing with high density networks means having hundreds or thousands of nodes sharing the limited bandwidth of the single frequency channel. Like other time scheduled protocols LMAC is not very well scalable with increasing nodes density. 
This means that due to higher contention in the neighborhood, as the number of the nodes in the network increases the chance to obtain a slot for them decreases. Therefore, the number of active nodes (nodes that control a slot) does not linearly increase as the density increases.

We adopt the definition of density $\rho$ used in [6]:

$$
\rho=\frac{N \pi r^{2}}{A}
$$

where $N$ is the number of nodes in the network, $A$ is the size of the area where the network is deployed, and $r$ is the transmission range of the nodes (nodes are supposed to have a circular radio coverage); $\rho$ defines the 1-hop density, i.e., the average number of nodes that a node with coverage range $r$ can reach with a broadcast.

To overcome the low scalability of LMAC and to make the protocol able to deal with high density, we propose to integrate a power control mechanism in the LMAC. The principle idea is to let the network dynamically decreases and re-sets up the transmission power of the nodes, in order to decrease node's local neighborhood density. The transceiver of the hardware we use has the capability to set the transmission power of the nodes to four different values. This means that the highest power available on the hardware at hand provides coverage range $R$, while the other power levels give coverage ranges respectively of $\frac{R}{2}, \frac{R}{4}$ and $\frac{R}{16}$. In the original implementation of the LMAC all the nodes have the same coverage range $R$ and they maintain it during whole their life time.

A proper coverage range for each possible topology and network density can be obtained if the total number of nodes in the network is known in advance before the LMAC starts. In this case, the original implementation of the LMAC could still well perform (in terms of number of active slots) in high density network. Our experimental results shown in Table I proves this issue. We have considered an area of $100 \times 100$ $m^{2}$, in which we deployed $N=100,200,300,400,500$ nodes and for each topology the transmission range has been chosen in a way to have a density of $\rho \simeq 7$, to assure that LMAC always gives the possibility to each node to control a slot. Unfortunately it is not always possible to decide offline in advance the optimal ranges for each possible scenario that could occur. Therefore, there is the need to find an adaptive and dynamic solution to determine the proper transmission range locally at each node and at run time during the slots allocation.

TABLE I

PERCENTAGE OF ACTIVE NODES IN LMAC PROTOCOL, IN CASE WE CHOSE OFFLINE THE PROPER RANGE THAT ASSURES TO HAVE A DENSITY $\rho \simeq 7$ FOR EACH TOPOLOGY.

\begin{tabular}{|c|c|c|}
\hline $\mathrm{N}$ & $\mathrm{R}(\mathrm{m})$ & active nodes $(\%)$ \\
\hline 100 & 16 & 100 \\
200 & 11 & 100 \\
300 & 9 & 100 \\
400 & 8 & 100 \\
500 & 7 & 100 \\
\hline
\end{tabular}

To cope with this problem and add power control to the LMAC, we propose addition of one slot per frame, which we call power slot. The other slots are called data slots, since they are used by nodes to forward the collected data. The power slot is used by nodes that can not find a free slot, in order to inform their neighborhood of their presence and of their need to find a data slot. The addition of only one slot per frame can not significantly degrade the performance of the protocol in terms of latency. There is no need to add more than one slot, even if the nodes that have found all the data slots as occupied are more than one. In fact, these nodes are not sending a data message in this particular slot, so eventual collisions are not critical. They only have to notify their neighborhood about their state. To do so they only have to send a signal and to occupy the wireless channel at the beginning of the power slot. All the nodes remain on at the beginning of the power slot to check the channel. If the channel is busy it means that one or more of node's neighbors are not able to find a free data slot to control. When a node finds the power slot busy it should apply one of the power control algorithms we propose in Sections III-A, III-B, and III-C.

We notice that the following algorithms could also be started without the need to add a slot per frame, by following a different policy. For example, a node could start the power control technique when it discovers, from the number of $\mathrm{CM}$ received during a frame, that the number of its neighbors is greater than a predefined threshold. This is an index of a high density network but this condition could not give a correct idea of the actual need to start the power control technique. In fact the density could be locally high but still enough to have all the nodes in the vicinity as active and no node in search of a free slot. In this case there would be no need to use the power control approach but the adopted decision condition would nevertheless perform it. Our approach avoids this critical point thanks to the addition of the power slot and the power signal sent by nodes in search for a slot.

\section{A. NoFreeSlot Approach}

In this approach, a node unable to find a free slot in its neighborhood broadcasts the proper signal in the power slot to its 1-hop neighbors. It then reduces its transmission power to the power level under its own level. For example, if the node hardware is set to the first power level, the highest one, it switches to the second level, therefore its transmission range reduces from $R$ to $\frac{R}{2}$. If the node hardware is set to the second power level it switches to the third level; therefore its transmission range reduces from $\frac{R}{2}$ to $\frac{R}{4}$ and so on. If the node is already transmitting with the lowest power level it cannot do anything else. In this case the protocol continues working as the original LMAC, i.e., the node periodically monitors the channel to find an empty timeslot. All nodes whose transmission power is not at the lowest level and have received the signal in the power slot reduce their transmission power too, by switching to the power level below their actual one. This power switching allows dynamically decreasing the local number of neighbors of each node.

\section{B. FreeSlot Approach}

In this approach, a node unable to find a free slot in its neighborhood broadcasts the proper signal in the power slot 
to its 1-hop neighbors and it reduces its transmission power to the power level under its own level. All the nodes that receive the signal in the power slot reduce their transmission power too by switching to the power level below their actual one, and also free their owned slot. Nodes that have released their slots fall again in the initial state of finding a free slot in the next frames. This slot liberation allows re-setting up the local network according to the new transmission ranges of the local nodes.

\section{FreeSlot and NoFreeSlot Second Order Approaches}

Both the two previous algorithms can also be extended to the 2-hop neighborhood.

As far as the NoFreeSlot concerns, the Second Order extension works as follows. A node unable to find a free slot in its neighborhood broadcasts the proper signal in the first half of the power slot to its 1-hop neighbors. It then reduces its transmission power to the power level under its own level. All the nodes that receive the signal in the power slot send a similar signal in the second part of the power slot to advertise to their the 2-hop away neighbors. They then reduce their transmission power by switching to the power level below their actual one. The 2-hops neighbors receive the busy channel signal in the second part of the power slot and they simply scale down their transmission power.

As far as the FreeSlot concerns, the Second Order extension works as follows. A node unable to find a free slot in its neighborhood broadcasts the proper signal in the first half of the power slot to its 1-hop neighbors and it reduces its transmission power to the power level under its own level. All nodes receiving the signal in the power slot send a similar signal in the second part of the power slot to advertise to their 2-hop away neighbors. Consequently they reduce their transmission power by switching to the power level below their actual one. Moreover, they free their own slot. The 2hops neighbors receive the busy channel signal in the second part of the power slot and they scale down their transmission power and they free their slot.

\section{Decision about the Sequential Operation}

In the previous description of algorithms, each node has to reduce its transmission power before it broadcasts the busy channel signal to its neighbors. It then reduces the power. This sequential choice could also be changed, by firstly reducing the power and then by notifying the neighbors. We will show in Section IV-A the impact of this sequential choice through the collected simulation results, even if is intuitive to understand that by firstly broadcasting and then reducing the power it is possible to advertise more nodes at the first attempt.

\section{E. Power Levels}

One should notice that in our proposed solutions the process of transmission power reduction does not have to be forced to stop when a node has a power level equal to the last one. It is possible to consider all the four available power levels by considering what we call SetOfRanges $=4$.
However, it also possible to consider only the first three levels (SetOfRanges $=3$ ) or the first two levels (SetOfRanges $=$ 2).

\section{EXPERIMENTAL RESULTS AND EVALUATIONS}

Results presented in this section have been obtained by implementing the LMAC original protocol and our proposed power controlled algorithms in a $\mathrm{C}++$ simulator. In the LMAC protocol it is possible to set up the frame duration and the number of slots per frame accordingly to the specific application requirements. In this paper the original LMAC implementation consists of a frame of 1 second and 32 data slots; power control LMAC versions have 32 data slots and one power slot. We simulate a static WSN composed of $N$ fixed nodes placed in a square area $A$; all the $N$ nodes are randomly deployed and uniformly distributed inside the area $A$. In order to simulate different network densities we consider topologies with different values of the parameter $N$ and the collected results have been obtained as an average on 200 different topologies for each value of $N$. We have verified that 200 topologies are enough to collect stable results. We suppose that at the beginning all nodes communicate with the same transmission range $R$.

We study the following performance metrics, (i) the percentage of active nodes as a function of the total number of nodes present in the network, and (ii) the latency required to accomplish the slot allocation investigated as the percentage of active nodes as a function of the time expressed in frames. The percentage of active nodes is calculated as:

$$
\text { ActiveNodes }(\%)=\frac{100 * \text { number of nodes owning time slot }}{N}
$$

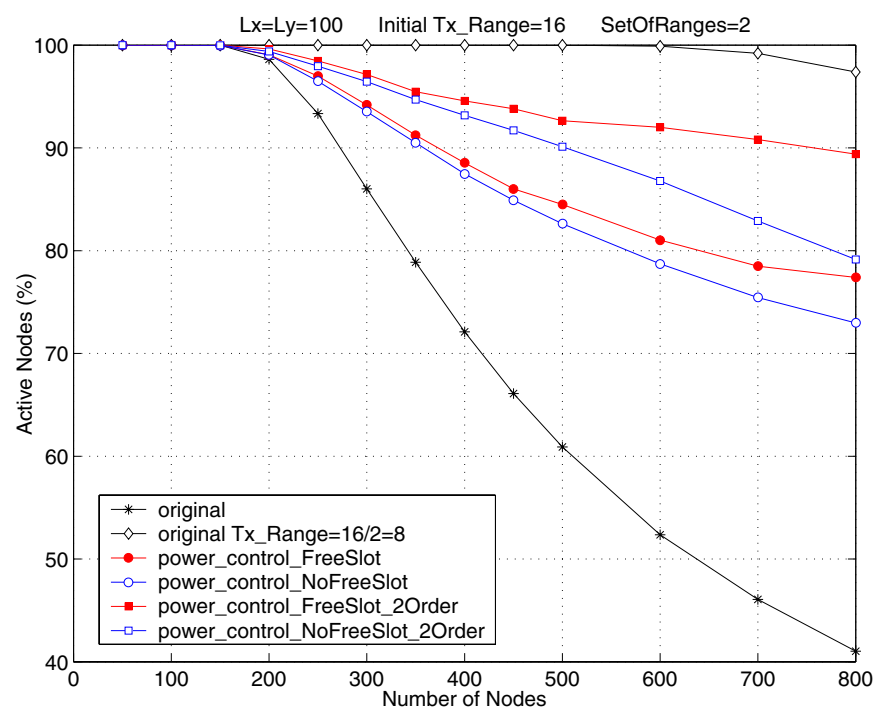

Figure 2. Power control algorithms firstly reduce the transmission range and then broadcast the power signal. Percentage of active nodes as a function of the number of nodes $N$ present in the network. Network parameter: $A=$ $100 x 100 m^{2}, R=16$, SetOfRanges $=2$. 


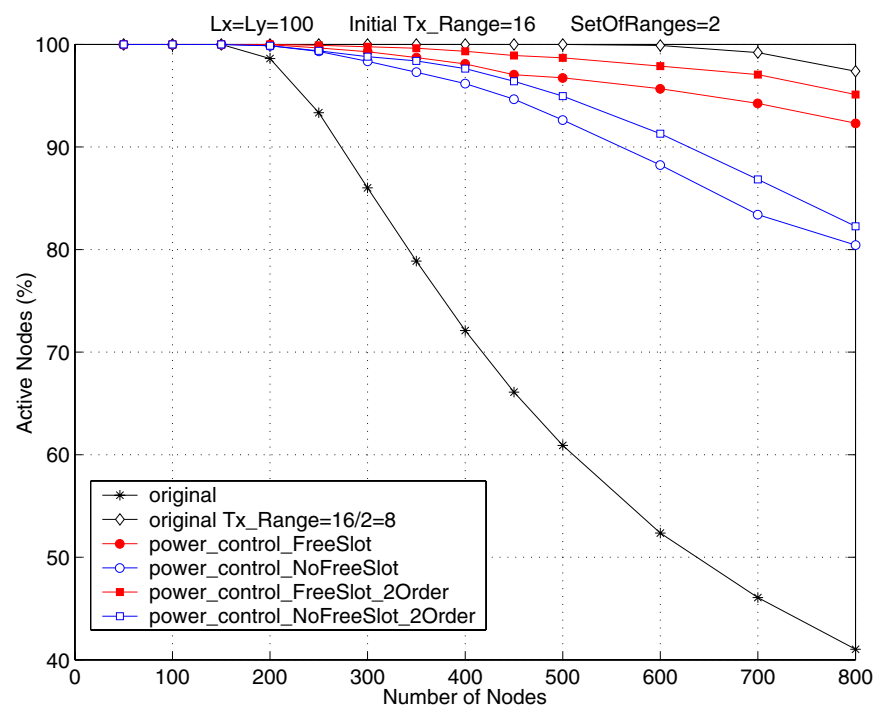

Figure 3. Power control algorithms firstly broadcast the power signal and then reduce the transmission range. Percentage of active nodes as a function of the number of nodes $N$ present in the network. Network parameter: $A=$ $100 \times 100 m^{2}, R=16$, SetOfRanges $=2$.

\section{A. Sequential Operation}

We start by investigating the impact of the sequential operation choice (as mentioned in Section III-D). In Figures 2 and 3 we refer to a network with parameters $A=100 x 100 \mathrm{~m}^{2}$ and $R=16 \mathrm{~m}$. Because of connectivity reasons, in a scenario with such a small initial range we implement the proposed algorithms by taking into account only the first two power levels. More transmission range reduction can lead to isolation of nodes and consequently loosing the network connectivity. We let the parameter $N$ to vary between 50 and 800 . These conditions allow studying network density $\rho$ respectively between 4 and 64 .

Figure 2 and Figure 3 plot the performance of the original LMAC protocol with $R=16 \mathrm{~m}$ with the power control versions with SetOfRanges $=2$. Figure 2 refers to the sequential order of firstly reducing the power and then broadcasting the power control message, while Figure 3 refers to the opposite sequential order. We also plot the curve related to the LMAC protocol, in case we should have chosen from the beginning the smaller range $R=8 \mathrm{~m}$. Original LMAC implementation with $R=8 \mathrm{~m}$ is a sort of upper bound since it represents the case in which all the $N$ nodes are working with the smallest range among the set of possible ranges (in this case SetOfRanges $=2$ ).

By comparing these two figures we can see that following the sequential order of firstly broadcasting the power control message and then reducing the power (Figure 3) gives better performances with respect to the opposite sequentiality of these two actions (Figure 2). Therefore in the rest of the paper we will only refer to the first option.

\section{B. Active Nodes}

We can observe from Figures 2 and 3 the inefficiency (in terms of number of active nodes) of LMAC protocol in dense networks. The percentage of active nodes decreases rapidly as the number of nodes $N$, and therefore the network density, increases. For $N \geq 300$ active nodes are always significantly below the $90 \%$.

In Figure 3 all the proposed power control algorithms perform better than the original protocol while facing high density networks, offering the opportunity to own a slot to a higher number of nodes. With NoFreeSlot approach the percentage of active nodes is always above $80 \%$, while with FreeSlot the percentage of active nodes remains over the $90 \%$ in all the studied topologies and it is very close to the upper bound. We conclude that the FreeSlot solution performs better than the NoFreeslot solution because it allows reorganizing the slot allocation in the neighborhood of the node that started the power control mechanism. The SecondOrder solutions allow improving the performances in the multihop scenarios. The gain achieved by the SecondOrder is much higher in the Figure 2, were firstly the transmission range is reduced. In that case the multihop behavior of the network is more evident.

TABLE II

DISTRIBUTION OF RANGES VALUES AFTER THE SINGLE HOP POWER CONTROL ALGORITHMS. NETWORK PARAMETERS: $A=100 x 100 \mathrm{~m}^{2}$, $R=16 m$.

\begin{tabular}{|c|c|c|}
\hline $\mathrm{N}$ & $\mathrm{R}$ & $\mathrm{R} / 2$ \\
\hline 100 & 100 & 0 \\
200 & 87 & 13 \\
300 & 50 & 50 \\
400 & 30 & 70 \\
500 & 22 & 78 \\
600 & 15 & 85 \\
700 & 10 & 90 \\
800 & 7 & 93 \\
\hline
\end{tabular}

We have also investigated the distribution of ranges values after applying the power control mechanism. We observe that the range distribution is affected by the single hop or second hop choices, but the results obtained from FreeSlot or NoFreeSlot approaches are quite similar. Concerning the single hop approaches we find the distribution reported in the Table II, where the second column reports the percentage of active nodes with range $r=R=16 \mathrm{~m}$ and the third column the percentage of active nodes with range $r=R / 2=8 \mathrm{~m}$.

In case of the second order approaches we find the results listed in Table III.

TABLE III

DISTRIBUTION OF RANGES VALUES AFTER THE SECOND HOP POWER CONTROL Algorithms. NetWork PARAMETERs: $A=100 \times 100 \mathrm{~m}^{2}$, $R=16 m$.

\begin{tabular}{|c|c|c|}
\hline $\mathrm{N}$ & $\mathrm{R}$ & $\mathrm{R} / 2$ \\
\hline 100 & 100 & 0 \\
200 & 77 & 23 \\
300 & 33 & 67 \\
400 & 19 & 81 \\
500 & 12 & 88 \\
600 & 8 & 92 \\
700 & 5 & 95 \\
800 & 3 & 97 \\
\hline
\end{tabular}




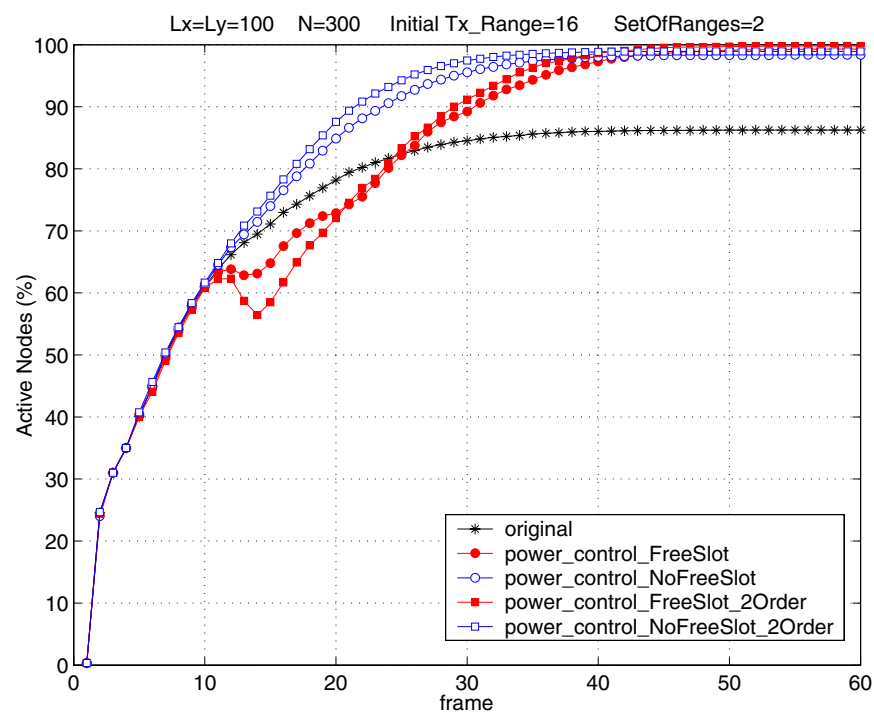

Figure 4. Latency. Network parameter: $A=100 \times 100 m^{2}, R=16$, SetOfRanges $=2, N=300$.

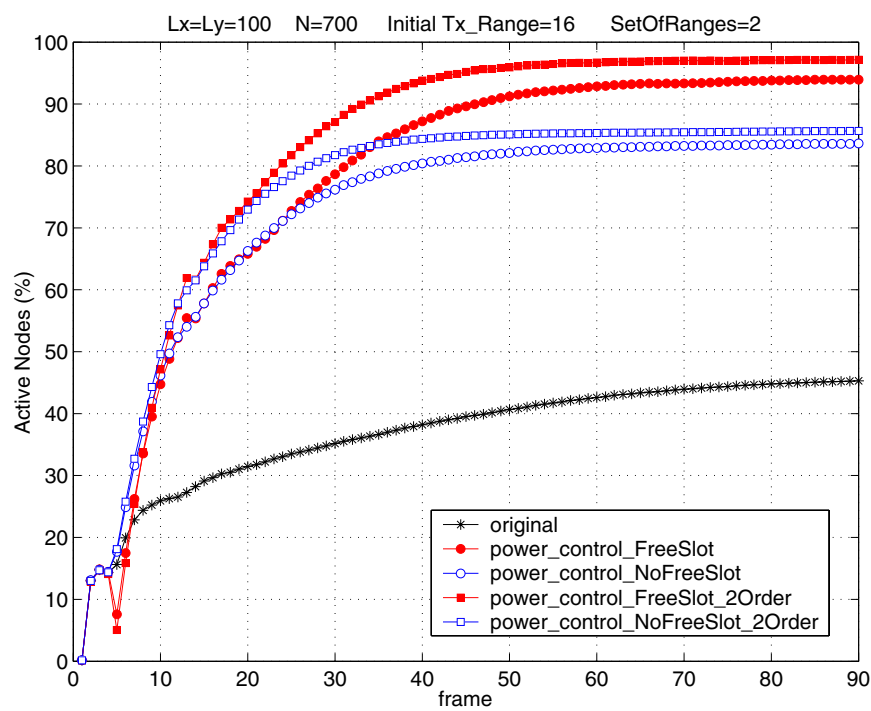

Figure 5. Latency. Network parameter: $A=100 \times 100 m^{2}, R=16$, SetOfRanges $=2, N=700$.

\section{Latency}

This performance improvement is achieved through a resetup of the network. This could negatively influence the latency, the time to wait in order to obtain a stable slot assignment. In order to evaluate the impact of the proposed power control solutions on the latency we study the time needed by the network to conclude the slot assignment and we compare it to the time needed by the original LMAC protocol. Figure 4 and Figure 5 plot the percentage of active nodes as a function of the time expressed in frames. In the first case we refer to a network with $N=300$ and in the second $N=700$. In the case of $N=300$ the power control solutions present higher latency than the original LMAC, but the difference between them is not too high: the latency is about 40 frames for the original LMAC, about 45 frames for the NoFreeSlot algorithms, and about 50 frames for the FreeSlot algorithms. Clearly the latency that we can support depends on the application the network is used for. Nevertheless, we notice that the difference between the original protocol and the power control ones is very small. When $N=700$ the power control solutions can even be more latency effective than the original protocol. In this case the latency of the original LMAC is about 80 frames, for the NoFreeSlot algorithms is about 60 frames and for the FreeSlot ones is about 80 frames. Among the power control solutions, obviously the NoFreeSlot presents lower latency compared to the FreeSlot, since the liberation of the slot from a subset of nodes needs more time to conclude the slot assignment. We can see nevertheless that the difference is not very high: when $N=300$ is almost of 5 frames while with $N=700$ the NoFreeSlot is almost 20 frames. We have focused the attention to these two particular topologies because they represent two different behaviors. When $N=300$ all the power control solutions show the same performance, therefore in this case the one with minor latency can be chosen. When $N=700$ a trade off has to be achieved: there is a clear gain in performance, of more than $10 \%$, by choosing the FreeSlot solution but this requires about 20 more frames to have a stable network.

\section{Power Levels}

In this subsection we focus the attention on the number of power levels (III-E). We show the performance, in terms of percentage of active nodes, obtained in other two scenario by implementing also the SetOfRanges $=3$ and Set $O f$ Ranges $=4$.

Figure 6 and Figure 7 refer to a scenario with an area of $A=$ $100 x 100 \mathrm{~m}^{2}$ and initial range $R=40$. In this case we evaluate the performance of the power control solutions both with SetOfRanges $=2$ and SetOfRange $=3$. The parameter $N$ varies between 50 and 500 , therefore the initial density $\rho$ of the network varies between 25 and 400. By comparing the results obtained using SetOfRanges $=2$ in Figure 6 with the ones using SetOfRanges $=3$ in Figure 7 we observe that in such a high density with the availability of three power levels the performance improvement is even bigger. With LMAC the percentage of active nodes when $N>150$ is less than the $50 \%$. With SetOfRanges $=2$ for each different value of $N$ the power control solutions achieve $30 \%$ more of active nodes with respect to LMAC. When SetOfRanges $=3$ the number of active nodes with NoFreeSlot approach is always above the $70 \%$ and with the FreeSlot approach is always above the $90 \%$. In both cases, SetOfRanges $=2$ and SetOfRanges $=3$, the FreeSlot approach is very close to the upper bound found in correspondence of the LMAC original protocol with starting range for all the nodes $R=40 \mathrm{~m} / 4=10 \mathrm{~m}$. FreeSlot solution proves to have a better performance compared to the NoFreeSlot solution, when we run the protocol with three available power levels. In both of these two figures we notice that there is almost no difference between the first order approaches and the second order ones, which is due to the particular scenario investigated. In other words, such an area 


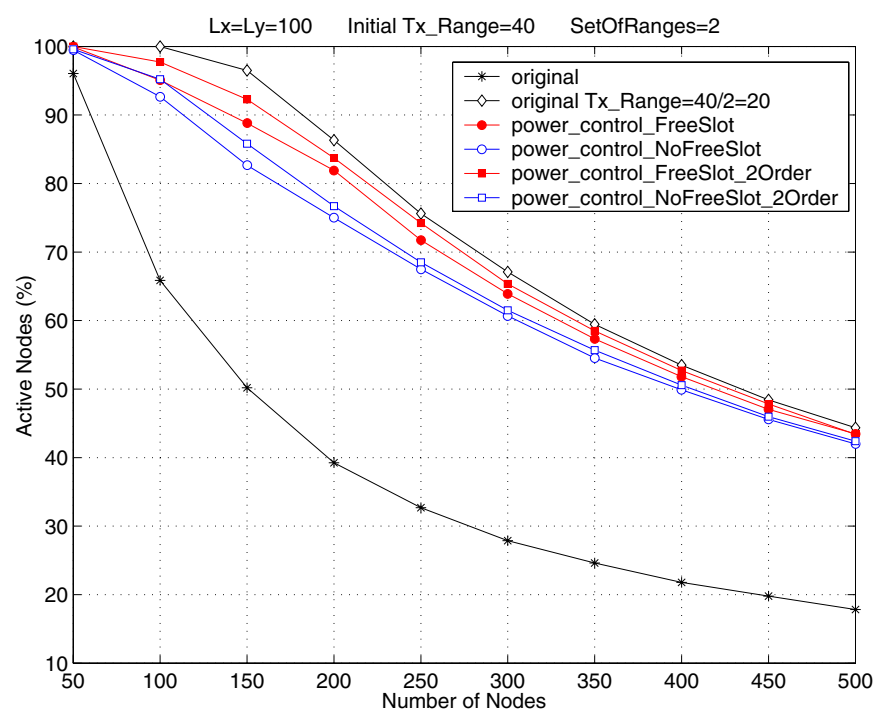

Figure 6. Percentage of active nodes as a function of the number of nodes $N$ present in the network. Network parameter: $A=100 x 100 \mathrm{~m}^{2}, R=40$, SetOfRanges $=2$.

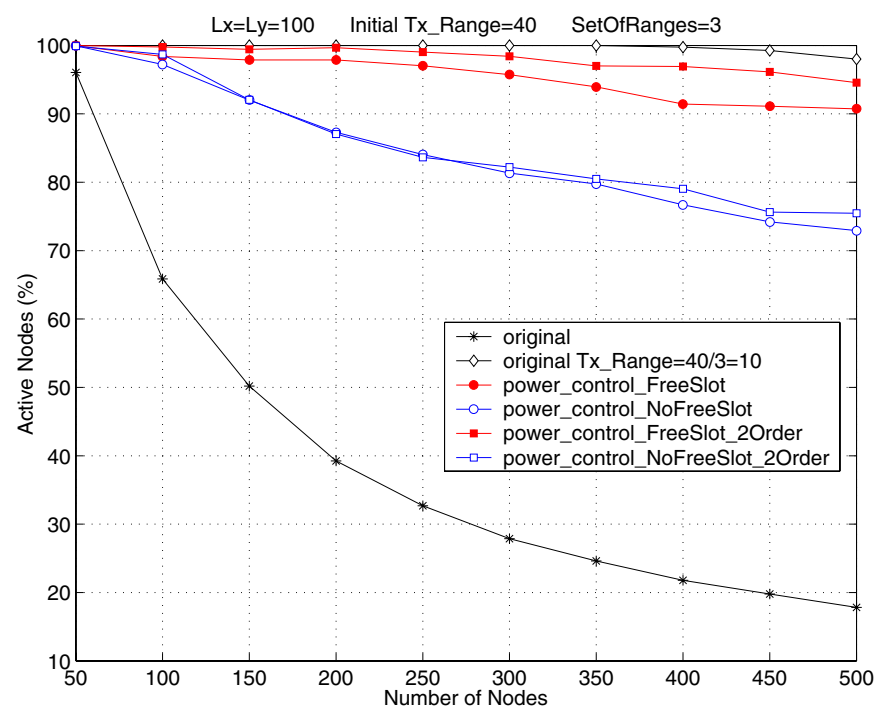

Figure 7. Percentage of active nodes as a function of the number of nodes $N$ present in the network. Network parameter: $A=100 x 100 \mathrm{~m}^{2}, R=40$, SetOfRanges $=3$

of $A$ and such an initial range of $R$ almost simulate a single hop network and therefore there is no need to use the second order extension.

Concerning the single hop approaches we find the distribution reported in the Table IV, where the second column reports the percentage of active nodes with range $r=R=40 \mathrm{~m}$, the third column the percentage of active nodes with range $r=R / 2=20 \mathrm{~m}$ and the last column the percentage of active nodes with range $r=R / 4=10 \mathrm{~m}$. In case of the second order approaches we find the range distributions reported in Table V.

The scenario shown in Figure 8 is even more dense and allows to exploit the benefits of the use of all the four
TABLE IV

DISTRIBUTION OF RANGES VALUES AFTER THE SINGLE HOP POWER CONTROL ALGORITHMS. NETWORK PARAMETERS: $A=100 x 100 \mathrm{~m}^{2}$, $R=40 m$.

\begin{tabular}{|c|c|c|c|}
\hline $\mathrm{N}$ & $\mathrm{R}$ & $\mathrm{R} / 2$ & $\mathrm{R} / 4$ \\
\hline 100 & 29 & 33 & 38 \\
200 & 7 & 17 & 76 \\
300 & 3 & 9 & 88 \\
400 & 2 & 7 & 91 \\
500 & 1 & 5 & 94 \\
\hline
\end{tabular}

TABLE V

DISTRIBUTION OF RANGES VALUES AFTER THE SINGLE HOP POWER CONTROL ALGORITHMS. NETWORK PARAMETERS: $A=100 x 100 \mathrm{~m}^{2}$,

$$
R=40 m \text {. }
$$

\begin{tabular}{|c|c|c|c|}
\hline $\mathrm{N}$ & $\mathrm{R}$ & $\mathrm{R} / 2$ & $\mathrm{R} / 4$ \\
\hline 100 & 14 & 45 & 41 \\
200 & 3 & 12 & 85 \\
300 & 2 & 7 & 91 \\
400 & 1 & 5 & 94 \\
500 & 1 & 3 & 96 \\
\hline
\end{tabular}

power levels. We refer to a network with an area of $A=$ $50 \times 50 \mathrm{~m}$ and initial range of $R=40$. The number of nodes $N$ varies between 50 and 500 , having therefore densities varying between 100 and 1000 . In this case there is obviously no need to use the second order extension. We concentrate only on the FreeSlot approach and we show in Figure 8 the gain that we obtain thanks to the power control, in the three cases SetOfRanges $=2$, SetOfRanges $=3$ and SetOfRanges $=4$. By using all the available power levels we can assure a performance of $100 \%$ of active nodes in all the studied topologies, while the original LMAC only can achieve performances of around $10 \%$ for number of nodes greater than $N=250$.

We report in the Table VI the range distribution in case of SetOfRanges $=4$, in which the second column reports the percentage of active nodes with range $r=R=40 \mathrm{~m}$, the third column the percentage of active nodes with range $r=R / 2=20 \mathrm{~m}$, and the fourth column the percentage of active nodes with range $r=R / 4=10 \mathrm{~m}$ and the last column the percentage of active nodes with range $r=R / 16=2.5 \mathrm{~m}$.

\section{RELATED WORK}

The issue of power control in WSN has been extensively studied. Algorithms such as [7], [8], [9] adjust the radio transmission power of each node in order to simultaneously

\section{TABLE VI}

DISTRIBUTION OF RANGES VALUES AFTER THE SINGLE HOP POWER CONTROL ALGORIthMS. NETWORK PARAMETERS: $A=50 x 50 \mathrm{~m}^{2}$, $R=40 m$.

\begin{tabular}{|c|c|c|c|c|}
\hline $\mathrm{N}$ & $\mathrm{R}$ & $\mathrm{R} / 2$ & $\mathrm{R} / 4$ & $\mathrm{R} / 16$ \\
\hline 100 & 2 & 8 & 20 & 70 \\
200 & 0.1 & 0.5 & 5 & 94.4 \\
300 & 0 & 0.5 & 3 & 96.5 \\
400 & 0 & 0.2 & 2 & 97.8 \\
500 & 0 & 0 & 1 & 99 \\
\hline
\end{tabular}




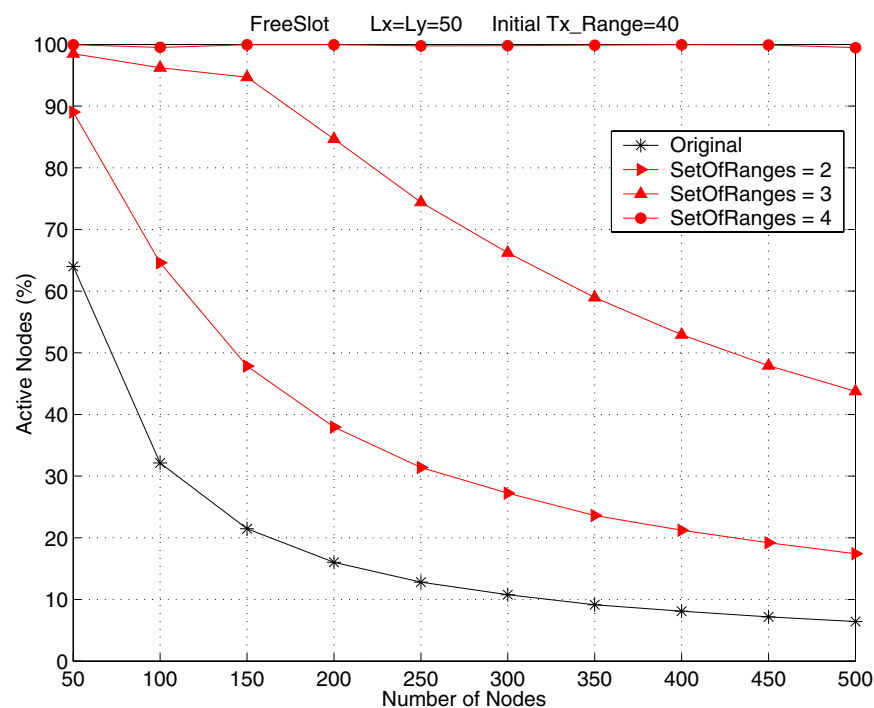

Figure 8. Percentage of active nodes as a function of the number of nodes $N$ present in the network. FreeSlot algorithm. Network parameter: $A=50 x 50 m^{2}, R=40$.

maintain a connected network and the node number of neighbors within a desired threshold.

Many proposals suggest transmission power control in order to achieve the optimal transmission power consumption for specified link qualities. Some centralized solutions adopt a proper power level for all the nodes of the network [10]. Other distributed solutions let each node choose its own transmission power [11]. Others instead fully exploit the configurable transmission power provided by radio hardware by supporting a packet level transmission power control [12].

\section{CONCLUSIONS AND Future WORKS}

We have addressed the effect of high density of wireless sensor network performance and have proposed a power control support for the LMAC protocol. By adding power control support to LMAC we significantly increased the number of active nodes (the nodes that control a timeslot) without affecting other performance metrics such as the latency. Our extensive experiments show that the power control proposals described in this paper efficiently improve the performances of the original LMAC protocol in very high dense networks.

The results that we have reported in this paper refer to the hypothesis of constant network density and static nodes inside the network. We do not consider here the possibility that the density can vary due to nodes mobility or battery failure; the only way that changes the density is the reduction of the transmission range decided by the power control algorithms. We extend our approach to include also increasing again the transmission power in case the network density falls below a certain threshold, to take into account node mobility or possible battery failure.

\section{REFERENCES}

[1] A. Mainwaring, J. Polastre, R. Szewczyk, D. Culler, J. Anderson, "Wireless Sensor Networks for Habitat Monitoring", WSNA 2002, 28 September, Atlanta, Georgia, USA.
[2] T. Gao, D. Greenspan, M. Welsh, R. R. Juang, A. Alm, "Vital Signs Monitoring and Patient Tracking Over a Wireless Network", IEEE EMBS 2005, 1.4 September, Shanghai, China.

[3] M. Coates, "Evaluating Causal Relationships Inwireless Sensor/Actuator Networks", ICASSP 2005, 18-23 March, Philadelphia, PA, USA.

[4] L. Yu, N. Wang, X. Meng, "Real-time Forest Fire Detection with Wireless Sensor Networks", WCNM 2005, September 23-26, Wuhan, China. Vol.2, pp. 1214-1217.

[5] L. van Hoesel, P. Havinga, "A lightweight medium access protocol (LMAC) for wireless sensor networks" In: Proc. of the $1^{\text {st }}$ Int. Workshop on Networked Sensing Systems (INSS), Tokyo, Japan, 2004.

[6] D. Estrin, R. Govindam, J. Heidemann, S.Kumar, "Next century challenges: scalable coordination in sensor networks", Mobicom 99, pp.263270.

[7] R. Ramanathan, R. Rosales-Hain, "Topology control of multihop wireless networks using transmit power adjustment” IEEE INFOCOM 2000, 26-30 March, Tel-Aviv, Israel.

[8] M. Kubisch, H. Karl, A. Wolisz, L. C. Zhong, J. Rabaey, ”Distributed algorithms for transmission power control in wireless sensor networks", IEEE WCNC 2003, 16-20 March, New Orleans, Lousiana, USA.

[9] D. Son, B. Krishnamachari, J. Heidemann, "Experimental study of the effects of transmission power control and blacklisting in wireless sensor networks", IEEE SECON 2004, 4-7 October, Santa Clara, CA, USA.

[10] S. Narayanaswamy, V. Kawadia, R. S. Sreenivas, P. R. Kumar, "Power Control in Ad Hoc Networks: Theory, Architecture, Algorithm and Implementation of the COMPOWProtocol", European Wireless Conference 2002, 25-28 February, Florence, Italy, pp.156162.

[11] M. Kubisch, H. Karl, A. Wolisz, L. C. Zhong, J. M. Rabaey, ’Distributed Algorithms for Transmission Power Control in Wireless Sensor Networks", WCNC 2003, 16-20 March, New Orleans, Lousiana, USA.

[12] S. Lin, J. Zhang, G. Zhou, L. Gu, T. He, J. A. Stankovic, "ATPC: Adaptive Transmission Power Control for Wireless Sensor Networks", SenSys 2006, 1-3 November, Boulder, Colorado, USA. 